\title{
Impact of delaying surgery after chemoradiation in rectal cancer: outcomes from a tertiary cancer centre in India
}

\author{
Praveen Kammar ${ }^{1}$, Aditi Chaturvedi ${ }^{1}$, Masillamany Sivasanker ${ }^{1}$, Ashwin de'Souza ${ }^{1}$, Reena Engineer ${ }^{2}$, \\ Vikas Ostwal $^{3}$, Avanish Saklani ${ }^{1}$ \\ ${ }^{1}$ Colorectal Division, GI Services, Department of Surgical Oncology, ${ }^{2}$ Department of Radiation Oncology, ${ }^{3}$ Department of Medical Oncology, Tata \\ Memorial Centre, Homi Bhabha National Institute, Mumbai, India \\ Contributions: (I) Conception and design: A Saklani; (II) Administrative support: A de'Souza, R Engineer; (III) Provision of study materials or patients: \\ A Saklani, A de'Souza; (IV) Collection and assembly of data: A Chaturvedi; (V) Data analysis and interpretation: P Kammar; (VI) Manuscript writing: \\ All authors; (VII) Final approval of manuscript: All authors. \\ Correspondence to: Dr. Avanish Saklani. Chief of Colorectal Division, GI services, Department of Surgical Oncology, Tata Memorial Centre, Homi \\ Bhabha National Institute, E Borges Road, Parel, Mumbai, India. Email: asaklani@hotmail.com.
}

\begin{abstract}
Background: Delaying surgery after chemoradiation is one of the strategies for increasing tumor regression in rectal cancer. Tumour regression and PCR are known to have positive impact on survival.

Methods: It's a retrospective study of 161 patients undergoing surgery after neoadjuvant chemoradiation (NCRT) for locally advanced rectal cancer (LARC). Patients were divided into three categories based on the gap between NCRT and surgery, i.e., <8, 8-12 and >12 weeks. Tumor regression grades (TRG), sphincter preservation, post-operative morbidity-mortality and survival were evaluated.

Results: Sphincter preservation was significantly less in $>12$ weeks group compared to the other two groups $(\mathrm{P}=0.003)$. Intraoperative blood loss was significantly higher in $>12$ weeks group compared to $8-12$ weeks group $(\mathrm{P}=0.001)$. There was no difference in major postoperative morbidity and hospital stay among the groups. There was no significant correlation between delay and TRG ( $\mathrm{P}=0.644)$. At Median follow up of 49.5 months the projected 3-year overall survival (OS) and disease free survival (DFS) were not significantly different among the 3 groups (OS: $79.5 \%$ vs. $83.3 \%$ vs. $76.5 \%$; $\mathrm{P}=0.849$ and DFS $50.4 \%$ vs. $70.6 \%$ vs. $62 \%$; $\mathrm{P}=0.270$ respectively)

Conclusions: Delaying surgery by more than 12 weeks causes more blood loss but no change in morbidity or hospital stay. Increased time interval between radiation and surgery does not improve tumor regression and has no effect on survival.
\end{abstract}

Keywords: Delayed surgery; radiation; rectal cancer

Submitted Sep 02, 2019. Accepted for publication Nov 06, 2019.

doi: 10.21037/jgo.2019.12.04

View this article at: http://dx.doi.org/10.21037/jgo.2019.12.04

\section{Introduction}

Colorectal cancer in Indian subcontinent occurs at a younger age and is associated with higher proportion of poorly differentiated and signet ring tumors (1). Higher rates of progression during chemoradiation (2) and poorer disease free survival (DFS) (compared to older patients) (3) are noted in young patients. Tumor regression grade (TRG) and pathological complete response (PCR) in patients undergoing neoadjuvant chemoradiation (NCRT) for locally advanced rectal cancer (LARC) is very well studied domain in the west and south-east Asia. TRG and PCR are good surrogate markers for prognostic outcomes (4). PCR may have a positive impact on survival related outcomes (5). Delaying surgery is one of the strategies of increasing tumor regression in order to achieve PCR. However, in younger patients of Indian subcontinent the delay in surgery to achieve more tumor regression has to be 
balanced with the risk of disease progression. While delay may increase down staging due to continued cell kill, it also increases tissue fibrosis which can increase the surgical difficulty and may ultimately add to morbidity. On the other hand, a significant down staging of tumor may lead to less extensive surgery, sphincter preservation and in select cases avoiding surgery altogether with wait and watch policy. These factors have a major impact on quality of life, bowel, bladder function and sexual function especially in younger patient population.

\section{Aims and objectives}

The aim of this study is to evaluate the impact of delaying surgery after NCRT in LARC. The outcomes were evaluated in terms of TRG, sphincter preservation, postoperative morbidity-mortality and survival outcomes.

\section{Methods}

This is a retrospective study based on a prospectively maintained database. All patients with LARC who underwent NCRT followed by total mesorectal excision (TME), between January 2013 and December 2014 were enrolled in the study. The study was approved by Institutional Ethics Committee (1746/IEC-II/10-2016) from Tata Memorial Centre, Mumbai, India. Informed consent was obtained from all the patients enrolled in the study. The data pertaining to patient and treatment details, pathology reports, perioperative outcomes and follow up were obtained from the database. The initial evaluation included thorough history and clinical examination, colonoscopy and biopsy, contrast enhanced computerized tomography (CECT) of the thorax and abdomen, magnetic resonance imaging (MRI) of the pelvis, complete blood work along with CEA levels. All the patients were discussed in multi-disciplinary team meetings to decide the treatment plan. All T3, T4, node positive tumors and tumors with threatened circumferential resection margin (CRM) underwent NCRT. The NCRT schedule comprised of 50.4 Gy radiation given over a period of 5 weeks with oral capecitabine chemotherapy. Disease status post NCRT was reassessed with MRI after 6 weeks' gap. Depending on the tumour extent patients underwent sphincter preserving or sacrificing surgery. The approach of open or minimal access was at the discretion of the surgeon. Those who progressed on NCRT were excluded and received further chemotherapy. The selected patients were subgrouped in to three categories depending on time interval between completion of NCRT and surgery, i.e., $<8$, $8-12 \&>12$ weeks. The reason for different time intervals between completion of chemoradiation and surgery were availability of operating rooms, long waiting lists and patient compliance. Most of the patients coming to our institution are from lower socioeconomic strata and have to travel long distances to get treatment. Hence it is difficult to match theatre availability with patients' logistics, thus resulting in variable time intervals. Pathological analysis was performed by a dedicated pathologist experienced in the field of colorectal cancer. Mansard scoring system was used to assess TRG where a score of 1 indicates complete regression and a score of 5 indicates no regression. The post-operative morbidity was assessed using Clavin Dindo scale. Morbidity of grade 3 and above was recorded for the first 30 post-operative days. All the patients underwent post-operative adjuvant chemotherapy. overall survival (OS) was defined as the time period between date of registration and date of last follow up or death. DFS was defined as the time period between the date of registration and date of recurrence.

\section{Statistical analysis}

Continuous variables are represented as mean \pm standard deviation and categorical variables as numbers and percentages. Chi square test was used for comparing categorical variables. Means were compared using ANOVA. Medians were compared using independent samples test. Post Hoc test was used depending on the homogeneity of the variance. $\mathrm{P}$ value of less than 0.05 was considered as statistically significant. The Spearman correlation test was used to investigate the relationship between continuous variables and intervals between NCRT and surgery. OS and DFS were calculated using Kaplan-Meier curves. Analysis was done using SPSS 20.0.

\section{Results}

There were 183 patients enrolled in the cohort. Twenty-two patients were excluded ( 6 patients defaulted, 14 patients had disease progression, 2 underwent surgery outside). Hence 161 patients were available for analysis. Patient and disease characters, treatment details are elaborated in Table 1.

Patient and tumor characteristics were comparable across three groups except the delay time and final pathological nodal status. Time interval between completion of NCRT and surgery varied from 4.29 weeks to 81.57 weeks with 
Table 1 Patients and tumor characteristics, treatment details and recurrences

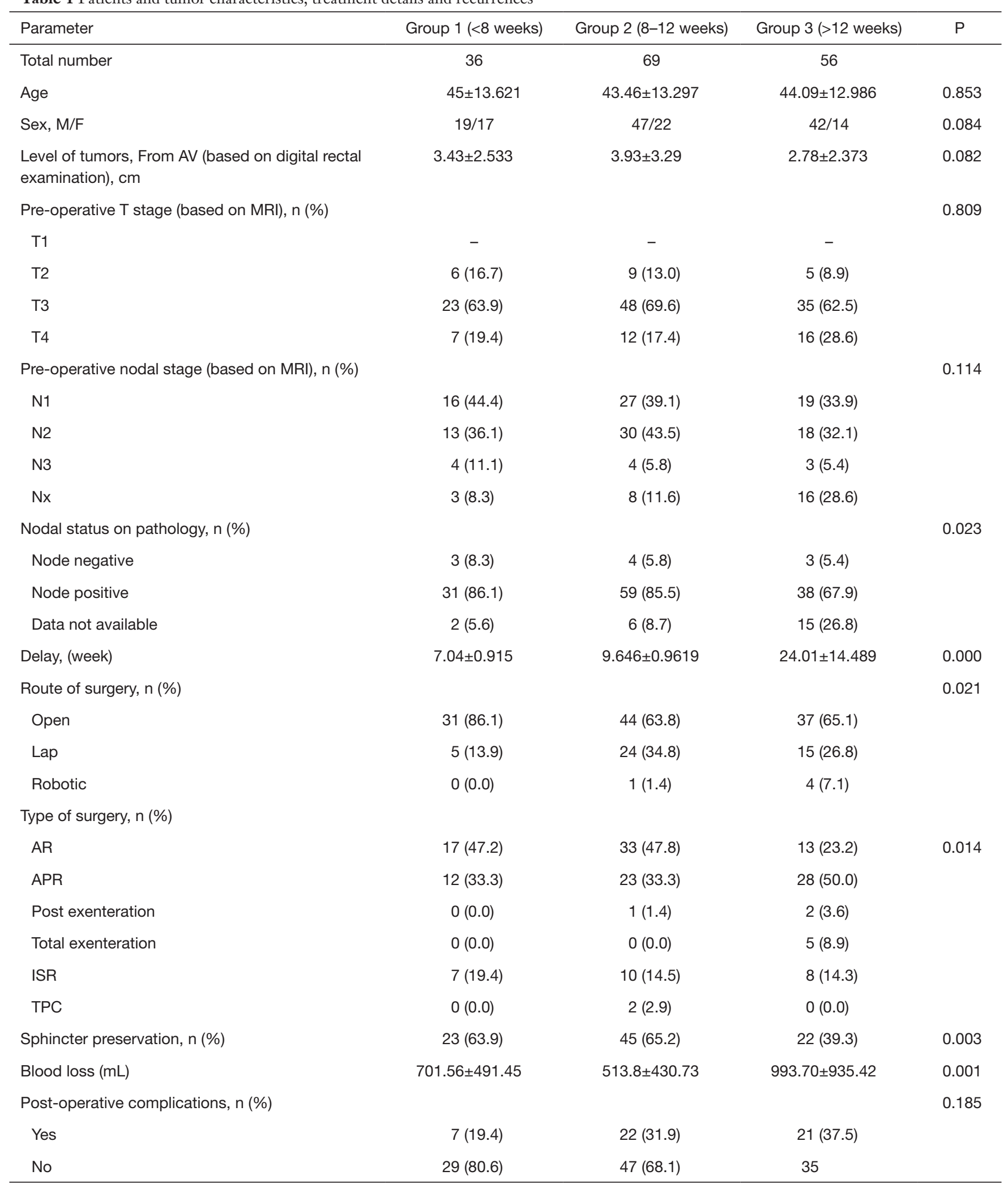

Table 1 (continued) 
Table 1 (continued)

\begin{tabular}{|c|c|c|c|c|}
\hline Parameter & Group 1 (<8 weeks) & Group 2 (8-12 weeks) & Group 3 (>12 weeks) & $\mathrm{P}$ \\
\hline 1 & 1 & 0 & 0 & \\
\hline 2 & 3 & 10 & 7 & \\
\hline 3a & 0 & 5 & 7 & \\
\hline $4 a$ & 1 & 0 & 0 & \\
\hline Not applicable & 29 & 47 & 35 & \\
\hline Median hospital stay (days) & $10.043 \pm 4.82$ & $9.63 \pm 3.127$ & $11.22 \pm 5.191$ & 0.203 \\
\hline Median follow up time (months) & $51.50 \pm 19.2$ & $52 \pm 18.3$ & $47 \pm 16.8$ & 0.231 \\
\hline Local & 4 & 5 & 3 & \\
\hline Systemic & 7 & 9 & 11 & \\
\hline Peritoneum & 1 & 2 & 2 & \\
\hline Local systemic & 1 & 2 & 1 & \\
\hline Nodes only & 0 & 0 & 1 & \\
\hline
\end{tabular}

$\mathrm{AV}$, anal verge; AR, anterior resection; APR, abdomino perineal resection; ISR, inter sphincteric resection; TPC, total proctocolectomy.

median of 10 weeks. The median time intervals among the three groups were 7.04, 9.646, 24.01 weeks respectively and the time differences among groups were significant $(\mathrm{P}=0.000)$.

There was no significant difference in tumor levels between the three groups $(\mathrm{P}=0.082)$. But there was considerable heterogeneity amongst the groups, as evidenced in Levine's test for homogeneity $(\mathrm{P}=0.015)$. Game's Howell's test showed a trend towards significant difference in tumor levels between 8-12 weeks group and $>12$ weeks group $(\mathrm{P}=0.065)$.

The number of patients undergoing open surgery was significantly higher in $<8$ weeks group compared to other two groups $(86.1 \%$ vs. less than $70 \%)$. Sphincter preservation was significantly less in $>12$ weeks group (50.0\% having abdomino perineal resection) compared to the other two groups $(39.3 \% v s$. more than $60 \%$, $\mathrm{P}=0.003$ ) and this is correlated well with much lower level of tumors in $>12$ weeks group. There was no data available on the total operative time. There was no qualitative analysis of surgical difficulty based on the waiting period. Intraoperative blood loss was significantly higher in $>12$ weeks group compared to $8-12$ weeks group $(\mathrm{P}=0.001)$. The hospital stay and complication rate were similar in three groups. Patterns of recurrence were also similar. Systemic recurrence was more common than local recurrences.

The comparison of pathological characteristics including TRG between groups is shown in Table 2. All the pathological parameters studies were comparable across the three groups.

TRG: 76 patients had TRG of $2(25.1 \%)$ or less $(24.1 \%$ had TRG 1); 135 patients (83.9\%) showed partial response; 18 patents each had stable disease $(11.2 \%)$. There was no significant correlation between delay time and TRG $(\mathrm{P}=0.644)$. There was no significant difference in TRG distribution across the three groups. Median TRG between the group was similar $(\mathrm{P}=0.792)$. Patients undergoing surgery after more than 12 weeks gap had significantly higher percentage of positive nodes compared to the other 2 groups $(\mathrm{P}=0.023)$. There was no data available about the toxicity of adjuvant chemotherapy and compliance rates.

Median follow up was of 49.5 [4-69] months. Median OS and DFS were not reached in the study cohort. The 
Table 2 Pathological characteristics between the groups

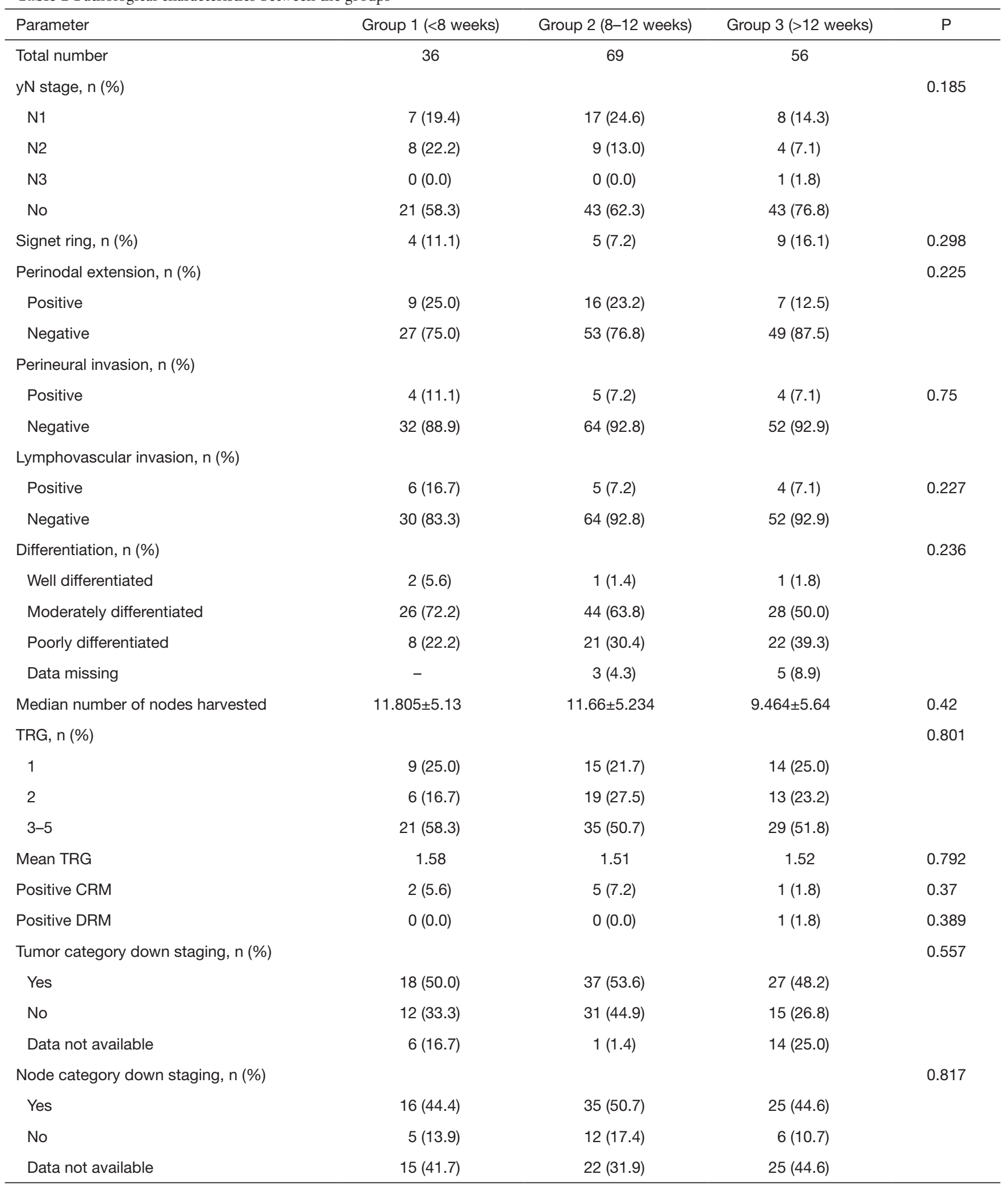

TRG, tumor regression grades; CRM, circumferential resection margin; DRM, distal resection margin. 
projected 3-year OS and DFS were not significantly different among the 3 groups (Table 3, Figure 1).

We also analyzed the long-term outcomes in terms of TRG and did not find any significant difference among different TRGs (Table 4, Figure 2).

\section{Discussion}

This study showed that delaying surgery after chemoradiation does not improve tumor regression. It also showed that both delay in surgery and tumor regression grades do not affect the outcomes. This study was retrospective but all three groups were fairly comparable. The cohort had a population

Table 3 OS and DFS according to groups

\begin{tabular}{lcc}
\hline Groups & $\begin{array}{c}\text { Projected 3-year DFS* } \\
(\%)\end{array}$ & $\begin{array}{c}\text { Projected 3-year OS** } \\
(\%)\end{array}$ \\
\hline$<8$ weeks & 50.4 & 79.5 \\
8-12 weeks & 70.6 & 83.3 \\
12 weeks & 62 & 76.5
\end{tabular}

*, $\mathrm{P}=0.270 ;{ }^{*}, \mathrm{P}=0.849$. OS, overall survival; DFS, disease free survival.

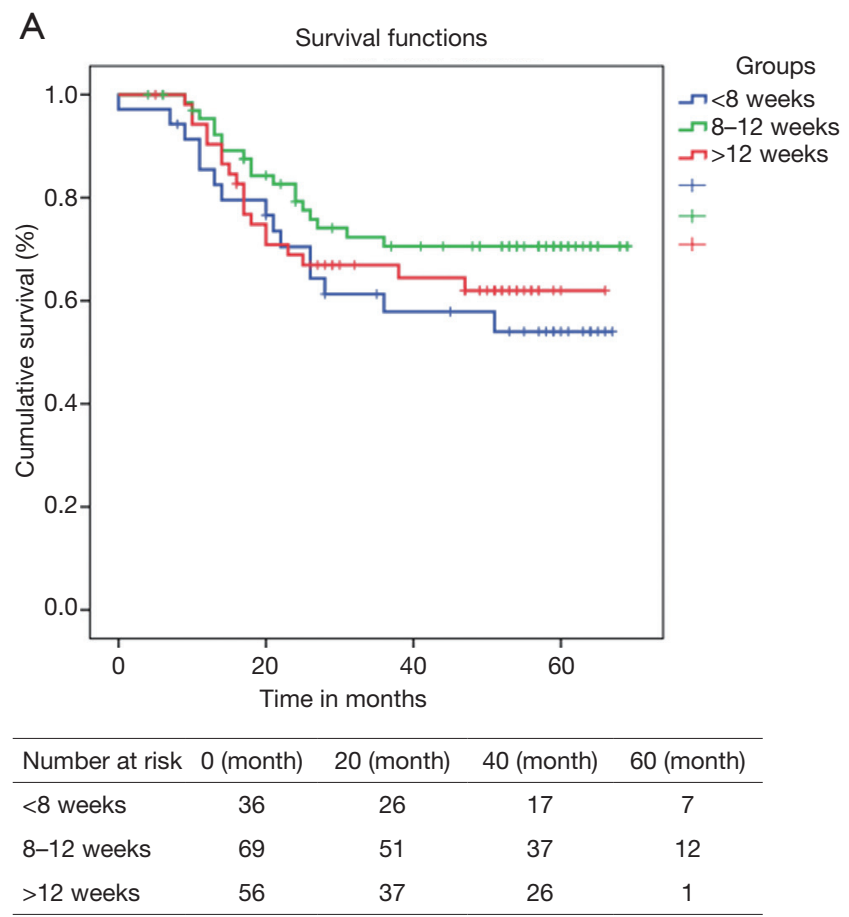

with median age of 44 years which is much younger than that reported in literature. The overall median age of patient population of 16 well selected studies analyzed in Martin et al.'s (5) meta-analysis was 60 years. Mean distance of tumor from anal verge in present cohort was $3.34 \mathrm{~cm}$. In the recent literature, only the GRECCAR 6 randomized controlled trial (6) had mean distance which was less than $4 \mathrm{~cm}$. In this aspect this patient cohort is unique.

The time interval between NCRT completion and surgery is a continuum unless actively controlled. This is one of the important technical glitches that is present in all the studies on this subject. Hence in the present analysis we compared whether the mean time gap in each group was significantly different than the others, so that we can ensure the results are meaningful. GRECCAR 06 is the only other study which has compared the time intervals of it's two study groups to ascertain that they are different (6).

Due to the continued cell kill induced by radiation, tumor down staging increases with time and PCR indicates the best outcome of radiation induced down staging. PCR has also been shown to improve survival $(5,7,8)$. In a recent meta-analysis of prognostic value of tumor regression, a significant association with OS benefit was observed in patients with PCR (9). Delaying surgery to achieve increased

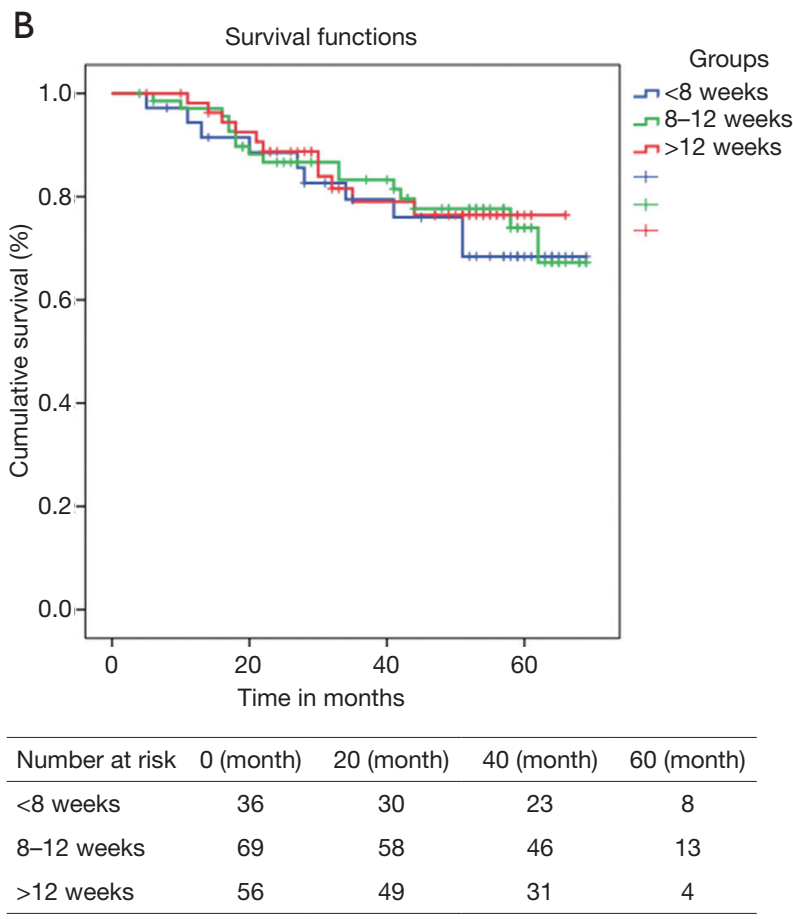

Figure 1 DFS (A) and OS (B) comparison between the three groups. DFS, disease free survival; OS, overall survival. 
PCR/down staging is one of the ways to improve outcomes in advanced rectal cancers. Lyon study proved that operating at 6-8 weeks showed significantly better PCR (10) and it became standard practice worldwide. Delay beyond seven weeks showed even higher PCR (11-13). Habr-Gama et al. evaluated waiting beyond 12 weeks and found that PCR significantly increased with longer wait. However there were no statistical differences in overall survival $(86 \% v s$. $81.6 \%)$ or DFS rates $(56.5 \%$ and $58.9 \%)$ between patients according to time interval ( $\leq 12 v s .>12$ weeks) (14). The only randomized trial with respect to delay beyond 6-8 weeks, GRECCAR 6, showed that waiting for 11 weeks did not

Table 4 Effect of TRG on OS and DFS

\begin{tabular}{lcc}
\hline TRG & 3-year DFS* & 3-year OS ${ }^{* *}$ \\
\hline 1 & 73 & 91 \\
2 & 71.8 & 83 \\
$3-5$ & 63.8 & 75.7 \\
\hline
\end{tabular}

*, $P=0.264$; **, $P=0.102$. TRG, tumor regression grade; OS, overall survival; DFS, disease free survival.

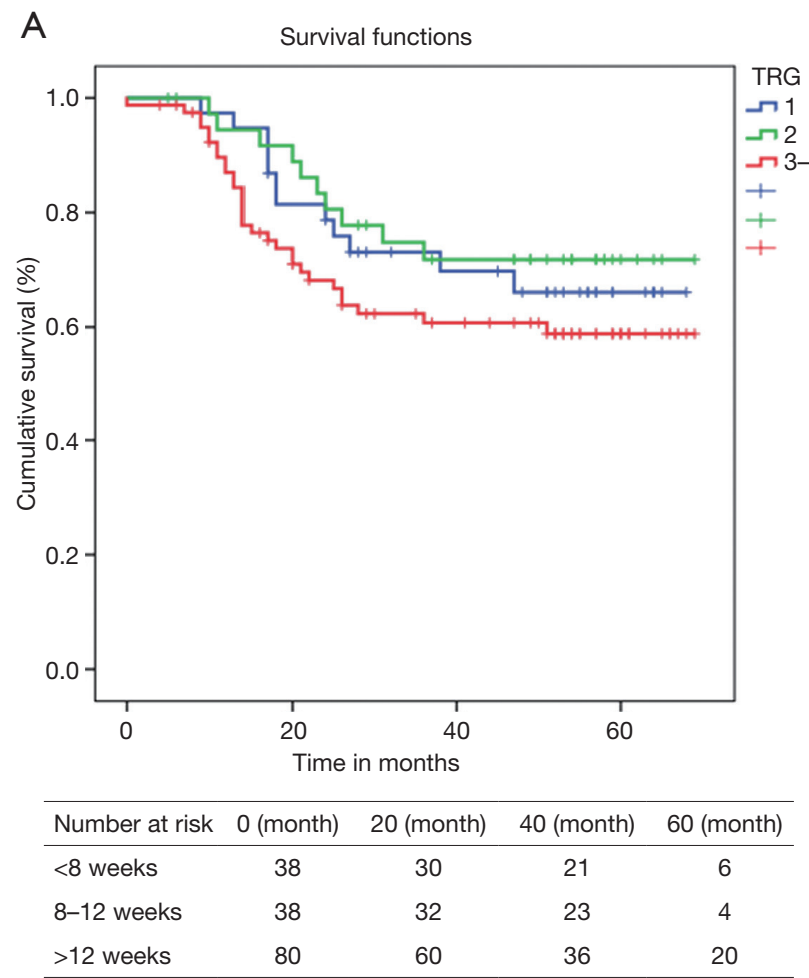

increase PCR rates further (6). Thus, indicating that an optimum waiting period lies between 6-11weeks and there is sealing effect for tumor response as far as waiting period is concerned. There are other studies which have shown that additional waiting may not be beneficial in terms of PCR $(15,16)$. The varied results of tumor regression with delayed surgery can be attributed to several factors. Majority of studies analyzing this subject are retrospective in nature with small numbers and also have heterogeneous patient population. But more than the statistical limitations, the varied results are also due to the complex interplay between pretreatment parameters and multiple other factors beyond delay after NCRT affecting the PCR and tumor regression. Non circumferential involvement (17-19), low pretreatment CEA (18-21), normalization of CEA after NCRT (22) were shown to be good prognostic factors while advanced $\mathrm{T}$ stage (23) and elevated CEA levels correlated with incomplete response (24). One study showed that tumors closer to anal verge had bad response to NCRT (24). Contradicting this fact Das et al. showed that distance of more than $5 \mathrm{~cm}$ from anal verge led to poorer response (19). Non-smokers, clinically node negative tumors, tumor

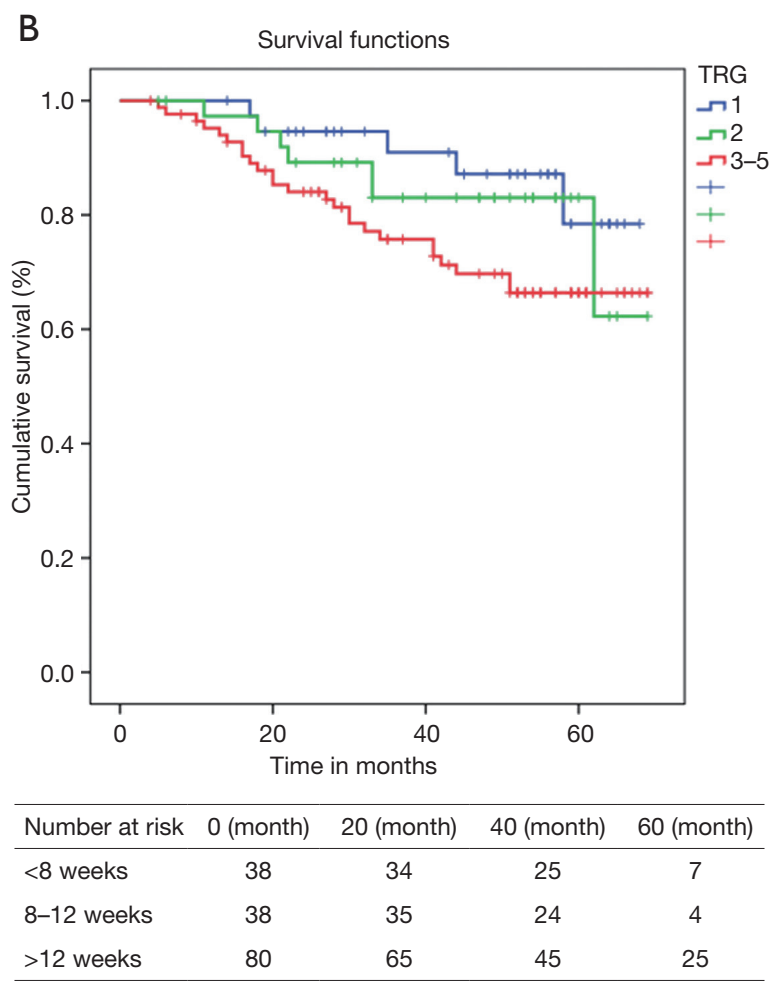

Figure 2 DFS (A) and OS (B) comparisons among different TRG scores. DFS, disease free survival; OS, overall survival; TRG, tumor regression grades. 
distance of more than $5 \mathrm{~cm}$, well-differentiated tumor, pre-NCRT CEA levels less than of $5 \mathrm{ng} / \mathrm{mL}$ and median interval to surgery of $>8$ weeks were independent clinical predictors for PCR in rectal cancer patients treated with long course of NCRT (25). Literature evidence is conflicting upon the influence of histology on tumor down staging. Signet ring was seen as a good factor by one study (17) while another study showed negative impact of signet ring histology (23) and yet another showed no influence of histologic subtype (26). In yet another meta-analysis, mucinous adenocarcinoma has been shown to have reduced rates of PCR and tumour downstaging following NACTRT with poorer overall survival following resection (27).

Down staging is expected to lead to more sphincter saving surgeries. However same has not been noted in several studies. This could be attributed to higher tumor locations in most of these studies $(10,11,13)$. Two Asian studies with mean distance from anal verge less than $5 \mathrm{~cm}$ showed no effect of delay on sphincter preservation $(15,16)$ and even GRECCAR 6 trial, with mean distance from anal verge close to $3 \mathrm{~cm}$ did not show any impact (6). But the present study showed significantly higher sphincter preservation in two groups where surgery was done less than 12 weeks. However, the group of $>12$ weeks had much lower tumors compared to the other 2 groups though it was not significant. Being a retrospective study selection bias would have pushed very low lying tumors for longer waiting periods in the hope of increased down staging.

Morbidity rates in the present series were comparable in all groups but blood loss was more in patients with delay more than 11 weeks. This could be an indirect indicator of surgical difficulty due to fibrosis. This is important considering the fact that more open surgeries in significant numbers happened in "less than 8 weeks" group. But we do not have the data on total operative time and qualitative details of surgical difficulty. Morbidity and complications were significantly higher in the 12 weeks group in GRECCAR6 trial (6) which were due to medical complications. A recent meta-analysis has reported no difference in complication rates depending on the waiting period (28).

Martin et al.'s meta-analysis showed that PCR led to good survival outcomes (5). The maximum gap analyzed here was 9 weeks. There were no RCTs in the meta-analysis. Compared to non-responders PCR patients had more than 3 -fold OS and DFS advantage. But the results cannot be extrapolated to all cases since these results were based on non RCT data, without significant low rectal tumors, nonuniform adjuvant therapy, mostly non-Asian patient cohort, and maximum delay of 9 weeks. Factors leading to PCR are not evaluated or discussed in this meta-analysis nor were the surgical outcomes (5). The more recent meta-analysis did not show any survival advantage of delaying surgery beyond the classical waiting period of 6-8 weeks in spite of showing $6 \%$ increase in PCR with longer waiting (28). In the present study analyzed there was no significant increase in TRG with longer waiting periods. Expectedly neither the TRG nor the delay period showed any impact on survival. Same results with respect to different waiting periods were seen in Lyon study after 17 year follow up, though there was significant increase in PCR with longer waiting period (4).

This is a study conducted in a tertiary cancer care center which handles one of the highest cancer burdens in the south east Asia. As a standard of care, all LARCs are treated by traditional long course CRT. The different delay times between NCRT and surgery occur due to several factors including triaging of patients due to long waiting lists, patients' preference and availability of operation rooms. This study was conducted to find out if delay was affecting the tumor regression and outcomes. Since the patient population is much younger with low rectal cancers, a better tumor response was expected to influence extent of surgery and functional outcomes. The results of the study, if significant, were expected to change the hospital policies as well; e.g., having a fixed time interval between NACRT and surgery and referring patients which cannot be accommodated in the specified time period to other centers. But the study results showed that in Indian population, increasing the waiting period may not increase the tumor regression and may increase surgical difficulty. There was no impact of tumor regression or increased waiting period on the survival outcomes. This is a retrospective study with all its inherent drawbacks. The details of the adjuvant treatment were not available. But the follow up was considerably long thus making these results meaningful at least in select patient sub group.

\section{Conclusions}

Delaying surgery beyond 8 weeks after NCRT in LARC does not improve the tumor regression. A delay beyond 12 weeks leads to more blood loss and possibly increases surgical difficulty but no change in morbidity or hospital stay was noted. Delaying surgery by more than 8 weeks does 
not impact survival. Further prospective studies with longer follow up are needed to throw more light on this issue in rectal cancer.

\section{Acknowledgments}

None.

\section{Footnote}

Conflicts of Interest: The authors have no conflicts of interest to declare.

Ethical Statement: The authors are accountable for all aspects of the work in ensuring that questions related to the accuracy or integrity of any part of the work are appropriately investigated and resolved. The study was approved by Institutional Ethics Committee (1746/IECII/10-2016) from Tata Memorial Centre, Mumbai, India. Informed consent was obtained from all the patients enrolled in the study.

\section{References}

1. Patil PS, Saklani A, Gambhire P, Mehta S, et al. Colorectal Cancer in India: An Audit from a Tertiary Center in a Low Prevalence Area. Indian J Surg Oncol 2017;8:484-90.

2. Shinde RS, Katdare N, Kumar NAN, et al. Impact of histological subtype on treatment outcomes in locally advanced rectal adenocarcinoma treated with neoadjuvant chemoradiation. Acta Oncol 2018;57:1721-3.

3. Pokharkar AB, Bhandare M, Patil P, et al. Young Vs Old Colorectal Cancer in Indian Subcontinent: a Tertiary Care Center Experience. Indian J Surg Oncol 2017;8:491-8.

4. Cotte E, Passot G, Decullier E, et al. Pathologic Response, When Increased by Longer Interval, Is a Marker but Not the Cause of Good Prognosis in Rectal Cancer: 17-year Follow-up of the Lyon R90-01 Randomized Trial. Int J Radiat Oncol Biol Phys 2016;94:544-53.

5. Martin ST, Heneghan HM, Winter DC. Systematic review and meta-analysis of outcomes following pathological complete response to neoadjuvant chemoradiotherapy for rectal cancer. Br J Surg 2012;99:918-28.

6. Lefevre JH, Mineur L, Kotti S, et al. Effect of Interval (7 or 11 weeks) Between Neoadjuvant Radiochemotherapy and Surgery on Complete Pathologic Response in Rectal Cancer: A Multicenter, Randomized, Controlled Trial
(GRECCAR-6). J Clin Oncol 2016;34:3773-80.

7. Yeo SG, Kim DY, Kim TH, et al. Pathologic complete response of primary tumor following preoperative chemoradiotherapy for locally advanced rectal cancer: long-term outcomes and prognostic significance of pathologic nodal status (KROG 09-01). Ann Surg 2010;252:998-1004.

8. Macchia G, Gambacorta MA, Masciocchi C, et al. Time to surgery and pathologic complete response after neoadjuvant chemoradiation in rectal cancer: A population study on 2094 patients. Clin Transl Radiat Oncol 2017;4:8-14.

9. Kong JC, Guerra GR, Warrier SK, et al. Prognostic value of tumour regression grade in locally advanced rectal cancer: a systematic review and meta-analysis. Colorectal Dis 2018;20:574-85.

10. Francois $Y$, Nemoz CJ, Baulieux J, et al. Influence of the interval between preoperative radiation therapy and surgery on downstaging and on the rate of sphinctersparing surgery for rectal cancer: the Lyon R90-01 randomized trial. J Clin Oncol 1999;17:2396.

11. Moore HG, Gittleman AE, Minsky BD, et al. Rate of pathologic complete response with increased interval between preoperative combined modality therapy and rectal cancer resection. Dis Colon Rectum 2004;47:279-86.

12. Kalady MF, de Campos-Lobato LF, Stocchi L, et al. Predictive Factors of Pathologic Complete Response After Neoadjuvant Chemoradiation for Rectal Cancer. Ann Surg 2009;250:582.

13. Tulchinsky H, Shmueli E, Figer A, et al. An interval $>7$ weeks between neoadjuvant therapy and surgery improves pathologic complete response and disease-free survival in patients with locally advanced rectal cancer. Ann Surg Oncol 2008;15:2661-7.

14. Habr-Gama A, Perez RO, Proscurshim I, et al. Interval between surgery and neoadjuvant chemoradiation therapy for distal rectal cancer: does delayed surgery have an impact on outcome? Int J Radiat Oncol Biol Phys 2008;71:1181-8.

15. Jeong DH, Lee HB, Hur H, et al. Optimal timing of surgery after neoadjuvant chemoradiation therapy in locally advanced rectal cancer. J Korean Surg Soc 2013;84:338-45.

16. Lim SB, Choi HS, Jeong SY, et al. Optimal surgery time after preoperative chemoradiotherapy for locally advanced rectal cancers. Ann Surg 2008;248:243-51.

17. Jayanand SB, Seshadri RA, Tapkire R. Signet ring cell histology and non-circumferential tumors predict 
pathological complete response following neoadjuvant chemoradiation in rectal cancers. Int J Colorectal Dis 2011;26:23-7.

18. Huh JW, Kim HR, Kim YJ. Clinical prediction of pathological complete response after preoperative chemoradiotherapy for rectal cancer. Dis Colon Rectum 2013;56:698-703.

19. Das P, Skibber JM, Rodriguez-Bigas MA, et al. Predictors of tumor response and downstaging in patients who receive preoperative chemoradiation for rectal cancer. Cancer 2007;109:1750-5.

20. Wallin U, Rothenberger D, Lowry A, et al. CEA - A Predictor for Pathologic Complete Response After Neoadjuvant Therapy for Rectal Cancer. Dis Colon Rectum 2013;56:859.

21. Lin JZ, Pan ZZ, Zeng ZF, et al. Multivariate analysis of clinicopathologic factors correlated with pathological complete response following preoperative radiotherapy in rectal adenocarcinoma. Ai Zheng 2009;28:919-22.

22. Kleiman A, Al-Khamis A, Farsi A, et al. Normalization of CEA Levels Post-Neoadjuvant Therapy is a Strong Predictor of Pathologic Complete Response in Rectal Cancer. J Gastrointest Surg 2015;19:1106-12.

23. Engineer R, Basu T, Chopra S, et al. Factors influencing response to neoadjuvant chemoradiation and outcomes

Cite this article as: Kammar P, Chaturvedi A, Sivasanker M, de'Souza A, Engineer R, Ostwal V, Saklani A. Impact of delaying surgery after chemoradiation in rectal cancer: outcomes from a tertiary cancer centre in India. J Gastrointest Oncol 2020;11(1):13-22. doi: 10.21037/jgo.2019.12.04 in rectal cancer patients: tertiary Indian cancer hospital experience. J Gastrointest Oncol 2015;6:155-64.

24. Restivo A, Zorcolo L, Cocco IMF, et al. Elevated CEA levels and low distance of the tumor from the anal verge are predictors of incomplete response to chemoradiation in patients with rectal cancer. Ann Surg Oncol 2013;20:864-71.

25. Bozkaya Y, Özdemir NY, Erdem GU, et al. Clinical predictive factors associated with pathologic complete response in locally advanced rectal cancer. J Oncol Sci 2018;4:5-10.

26. Shinde RS, Bhamre R, Niyogi D, et al. Does histology dictate outcomes of locally advanced rectal adenocarcinoma with complete pathological response following neoadjuvant chemoradiation? J Radiat Oncol 2018;7:85-9.

27. McCawley N, Clancy C, O’Neill BDP, et al. Mucinous Rectal Adenocarcinoma Is Associated with a Poor Response to Neoadjuvant Chemoradiotherapy: A Systematic Review and Meta-analysis. Dis Colon Rectum 2016;59:1200-8.

28. Petrelli F, Sgroi G, Sarti E, et al. Increasing the Interval Between Neoadjuvant Chemoradiotherapy and Surgery in Rectal Cancer: A Meta-analysis of Published Studies. Ann Surg 2016;263:458. 